\title{
ESTILOS PARENTALES, PARENTALIDAD POSITIVA Y FORMACIÓN DE PADRES
}

\section{PARENTING STYLES, POSITIVE PARENTING AND PARENTS FORMATION}

\author{
Álvaro Capano \\ Universidad Catolica del Uruguay \\ Asociación Civil SOMOS * \\ Andrea Ubach \\ Universidad de la República, Uruguay \\ Asociación Civil SOMOS
}

\begin{abstract}
Resumen: El presente trabajo surge como una inquietud de los autores, (integrantes de una ONG que se dedica al trabajo con niños y adolescentes que han sufrido la vulneración de algunos de sus derechos) por revisar en un rápido recorrido los desarrollos conceptuales que nos parecen más relevantes sobre los estilos educativos parentales y las posibles consecuencias del ejercicio de uno u otro en niños y/o adolescentes. Además, el trayecto realizado nos ha permitido reflexionar sobre algunos conceptos, dejar planteadas algunas preguntas y pensar sobre concepciones educativas alternativas, para llegar a la conclusión en última instancia de la relevancia que adopta la formación de padres, no como una posibilidad remota y aislada, sino como un camino necesario a recorrer por instituciones educativas, sociales y sanitarias, públicas y privadas.
\end{abstract}

Palabras Claves: estilo educativo parental, formación de padres

\begin{abstract}
This work comes as a concern of the authors - who belong to an NGO dedicated to working with children and adolescents who have suffered the violation of some of their rights- for primary review of those conceptual developments that seem most relevant to parenting styles and the possible consequences of the exercise of the different styles in the development of children and / or adolescents. In addition, this work has allowed us to reflect on some concepts, pose questions and think about alternative educational concepts. We managed to identify the crucial role of parent training, not as a remote and isolated possibility, but as a necessary path to be followed by public and private educational, social and health institutions.
\end{abstract}

Keywords: parental educational style, parent training

*SOMOS es una Asociación Civil de Montevideo-Uruguay especializada, dedicada a la asistencia psicológica, social y jurídica, en forma individual o familiar, de personas en situación de abuso y maltrato.

\section{Introducción}

Todo padre, madre o quien se encuentre en su lugar, sin duda, pretenden ser correctos con sus hijos, cumplir adecuadamente con su rol y ejecutar acertadamente sus funciones parentales. Es claro que ser padre o madre lleva por momentos a vivir altos niveles de satisfacción y beneplácito, no obstante esto, también existen en otras situaciones importantes niveles de tensión y sufrimiento, experiencias que generan frustración, renuncias e insatisfacciones (Máiquez, Rodrigo, Capote y Vermaes, 2000). Hay padres que han atravesado situaciones difíciles de sobrellevar como corolario de la relación con sus hijos y en esos momentos tal vez no supieron como resolverlas, quizás no hayan contado con la ayuda necesaria para afrontar dichos desafíos, que van desde pequeñas situaciones de la vida cotidiana a otras muy estresantes y dolorosas. Sin duda la tarea es complicada, no sirve la improvisación y se requiere en muchas oportunidades de destrezas específicas para afrontar los desafíos (Torío López, Peña Calvo y Rodríguez Menéndez, 2008). De haber recibido algún tipo de ayuda en esos momentos de duda, confusión, angustia ¿Qué y cuántas cosas podrían haber hecho de otra manera?

En este momento histórico y cultural ser padre y madre, coloca a las personas frente a una gran responsabilidad y las enfrenta a un gran desafío: la responsabilidad sin duda es la de educar, 
interviniendo, guiando, orientando, influyendo, mostrando, posibilitando la incidencia constante y regulada durante la evolución de la persona, con el objetivo de potenciar y optimizar su desarrollo y madurez, generando entonces las condiciones indispensables para el desarrollo saludable de los niños, niñas y adolescentes (Aguilar Ramos, 2002a; Comellas, 2003; 2009). Si bien los padres son los primeros responsables de la educación de sus hijos (Parada, 2010) es claro que no es dominio exclusivo de ellos el guiar, influir y educar a sus hijos, es una tarea compartida, encomendada a distintos grupos o entidades sociales (Comellas, 2009).

Vivimos actualmente en una sociedad donde se producen cambios a un ritmo vertiginoso, con transformaciones científicas, comunicacionales, tecnológicas (Aguilar Ramos, 2002a) y en dónde (aquí viene el desafío) los estilos educativos parentales y las formas de relación adulto-niño/adolescente con la que fueron educados los adultos en su infancia y adolescencia se encuentran, obsoletas, en franca declinación, ya no les sirven para educar a sus hijos (Aguilar Ramos, 2002a). En este sentido, ser padres es una tarea que se encuentra en permanente transformación, producto de cambios temporales, histórico-sociales y evolutivos a medida que cambia la edad de los padres y de los hijos. No es lo mismo ser padres en las décadas del 60 o 70 que serlo en los 90 (Máiquez et al., 2000). En términos generales los adultos de hoy nacidos en la segunda mitad del siglo pasado, al menos hasta mediados de los 70 (hoy padres y madres de niños y/o adolescentes) posiblemente hayan sido educados desde otro paradigma (Kuhn, 1962/1988), en donde el niño/adolescente era visto como objeto, que debía cumplir con su educación, ser obediente, dependiente y servil. Muchos de ellos entonces, han sido educados en el respeto sin condiciones a sus adultos, la obediencia irrestricta, donde la última palabra la tienen los mayores y además no se cuestionan las decisiones tomadas por los padres (DeMause, 1994). Era una época en la que prevalecía aún un modelo educativo parental, rígido, férreo, sin concesiones, o como lo denominara Baumrind (1966) autoritario.

Hoy la forma de relacionarse entre padres e hijos claramente es otra, el lugar, la consideración que se tiene por el niño y el adolescente en nuestra sociedad ha cambiado, las ideas y las acciones sobre éstos son distintas. Seguramente la lucha de más de 70 años por la reivindicación de los derechos de niños, niñas y adolescentes y el advenimiento de la Convención de los Derechos del Niño ( ([CDN] Asamblea General de las Naciones Unidas 1989) entre otras causas, han influido en el cambio de paradigma. La CDN es el documento de derechos humanos con más ratificaciones en el mundo y que recoge por primera vez en el mismo texto, derechos civiles, políticos, económicos, sociales y culturales. Es de tal importancia la transformación que produjo su aparición que García Méndez (1998) nos relata que la CDN es para los niños/as y adolescentes como la revolución francesa pero con 200 años de atraso. Aparecen entonces conceptos como el interés superior del niño, el niño como sujeto de derecho, etc. Son términos, conceptos que colocan a los niños/adolescentes en relación a los derechos humanos en igualdad de condiciones con los adultos.

Ahora, en el escenario actual, la principal dificultad que se les presenta a los padres, a la hora de ejercer las funciones parentales, es desde que estilo educativo parental se relacionan con su hijo/a. ¿Cómo educar? ¿El estilo que han interiorizado ellos en su infancia y adolescencia se encuentra en consonancia con los tiempos que corren? (Máiquez et al., 2000) ¿Se encuentra acorde a las necesidades de su hijo/a? (Aguilar Ramos, 2002a) ¿Qué cosas deberían tener en cuenta?

Es allí donde, en muchas ocasiones, se produce el distanciamiento, la fractura relacional entre padres e hijos. Nos encontramos entonces y sólo a modo de ejemplo, con algunos padres y madres con un estilo educativo con características rígidas, inflexibles, alejado de las necesidades de los chicos, autoritario. Otros padres y madres que han dimitido del ejercicio de sus funciones parentales, han renunciado al ejercicio de la autoridad, del rol protagónico que todo padre y madre debería tener en la educación de sus hijos, ejerciendo una disciplina "light" o "diluida" (Hernández Prados y López, 2006). Son adultos estos que ejercen sus funciones desde un estilo educativo permisivo o negligente (Maccoby \& Martín, 1983) contemplativo y por momentos pasivo.

Los estilos brevemente reseñados se encuentran dentro de una más extensa clasificación de estilos educativos parentales, sin embargo son una muestra clara de las dificultades con las que se encuentran los adultos a la hora de relacionarse con sus hijos y nosotros 
como profesionales en el trabajo con ellos. La cuestión vital en este momento es la de ayudar a los padres y madres a encontrar una forma adecuada de relacionarse y educar a sus hijos, un estilo educativo que acompase los tiempos que corren, intentando adaptarse y adoptar una forma que contemple las características y las necesidades de los niños y adolescentes, teniendo como parte central del modelo el ser afectuoso, respetuoso, tolerante, posibilitando el diálogo que aporte a la construcción, sin dejar de considerar la importancia que tienen los límites en la constitución del individuo y de las relaciones familiares/sociales.

Hay suficiente literatura sobre estilos educativos parentales, la intención de éste trabajo es recorrer algunos de los aportes que a nuestro entender resultan más interesantes, para luego intentar abordar la forma de educación parental que nos parece más atractiva para adoptar en los tiempos que vivimos.

Sin duda alguna, éste camino de ser padres e hijos comienza en el momento preciso en el que dos adultos deciden volverse padres y persiste luego, durante toda la vida. La influencia que ejerce la familia (padres, madres y otros) es de vital importancia en el desarrollo de los niños y niñas. A este primer momento se lo conoce como socialización.

\section{Proceso de socialización}

La socialización (Musitu y Cava 2001, p.115) "es el proceso mediante el cual las personas adquirimos los valores, creencias, normas y formas de conducta apropiados en la sociedad a la que pertenecemos". El proceso de socialización es el resultado de la interacción que se da entre el individuo y la sociedad. Es en ese proceso que se aprenden las pautas de comportamiento, las normas, roles y costumbres (Gervilla, 2008).

Según Musitu y Cava (2001), el periodo de la infancia y adolescencia es fundamental en este proceso de socialización, es por ello que la familia juega un papel privilegiado para la transmisión de pautas culturales. Tradicionalmente la socialización se pensaba en una sola dirección, desde los padres hacia los hijos (Bersabé, Fuentes y Motrico, 2001). En ese sentido son los padres quienes poseen, por experiencia, edad, razones sociales, biológicas y afectivas, el conocimiento para transmitir a sus hijos, valores, costumbres, sentimientos, pautas culturales y sociales. Desde el principio los padres cuentan con ideas preconcebidas, teorías implícitas, creencias y expectativas de cómo quieren criar y educar a sus hijos, acerca de las normas, ideas y valores que pretenden trasmitirles, de como deben ser los niños y de que forma educarlos. Sin embargo al nacer el niño/a puede ocurrir que las expectativas o ideas preconcebidas por los padres no se alcancen. Por lo tanto deben adecuar sus expectativas a la realidad y esto va ejerciendo una importante influencia en la forma que va tomando cada proceso de socialización en las diferentes familias (Musitu y Cava, 2001). También los hijos muchas veces influyen profundamente y sin saberlo en las vidas de sus padres y pueden funcionar como fuertes promotores del cambio en la vida de estos. Pudiendo llegar los padres a dejar el alcohol, el tabaco $\mathrm{u}$ otras sustancias adictivas (Musitu y Cava, 2001). El nacimiento de un niño, como hecho trascendente en el ciclo vital familiar, significa un cambio relevante en la vida familiar y en los integrantes de la misma. Es muy probable que los padres luego del nacimiento se planteen nuevas prioridades (González Tornaría, 2007).

Ya desde hace algún tiempo se considera que el proceso de socialización es bidireccional, también los padres son socializados por los hijos a lo largo de toda la vida (Ceballos y Rodrigo, 1998; Palacios, 1999; Musitu, Buelga, Lila y Cava, 2001; Musitu y Cava, 2001; Oliva, Parra y Arranz, 2008). A medida que van creciendo también los hijos influyen en los padres a través de otros agentes socializadores, como lo son los amigos, la escuela, los medios de comunicación, las modas, el mundo de Internet y las experiencias que ellos traen del medio externo y las vuelcan en sus familias. Los hijos introducen a los padres en el manejo de las nuevas tecnologías, exhiben y modelan nuevas formas de hablar y pensar (González Tornaría, 2007).

Según lo desarrollado hasta el momento, los espacios que rodean al sujeto, (niño o adolescente), la familia, el grupo de amigos, de compañeros, las personas significativas, inclusive el sistema educativo, juegan un papel trascendente, ejercen poderosas influencias sobre su desarrollo (Bronfenbrenner, 1987).

En ese sentido el modelo sociocultural propuesto por Vigotsky (1979) refleja cómo las personas cercanas, no solo de forma física sino también afectiva a los niños/as, son quienes los dirigen, los impulsan a avanzar en el aprendizaje de nuevos significantes. El desarrollo cognitivo 
se da en situaciones en donde el niño se lanza a resolver problemas siendo guiado por un adulto que prepara, modela, la solución más pertinente. Se estimula entonces el desarrollo cognitivo transportando conocimientos, capacidades, enseñanza de destrezas, de quienes la poseen -los padres-, aunque pueden ser otros adultos o incluso compañeros de clase, etc. de quienes las adquirirán. El desarrollo cognitivo entonces, surge a partir de las interacciones del niño con sus padres, (maestros, expertos) en donde se ven enfrentados a actividades para las que no poseen los conocimientos básicos y necesarios, por tanto requieren del apoyo de sus progenitores o figuras significativas, los cuales les permiten avanzar en el conocimiento, logrando la concreción de la tarea, convirtiéndose en una cadena secuencial en el trayecto de la vida, donde cada experiencia probablemente requerirá nuevos avances en su zonas de desarrollo (zdp).

Por su parte Rogoff (1993), en relación al conocimiento y otras actividades considera al contexto como algo inseparable de las acciones humanas, manifiesta la necesidad de tener en cuenta para el desarrollo infantil la mutua implicación que se ejerce entre los niños y el mundo social. Plantea que para el aprendiz (niño/a) tiene un valor trascendente la presencia, el acompañamiento y el estímulo de un otro, el individuo aprende, incorpora el conocimiento a través del contacto social, construye un puente entre lo que sabe y lo que va a aprender a nivel escolar. La institución educativa colabora en la resolución de problemas aportando instrumentos y tecnologías. El niño/a incorpora puntos de vista y la comprensión de otros. Toma entonces la responsabilidad de sortear los obstáculos que se le presenten, intenta soluciones, de esta forma promueve el desarrollo cognitivo. El progreso cognitivo del niño, evoluciona en el contexto de las relaciones sociales a este proceso la autora lo denominó "participación guiada". El concepto de participación es inseparable del de aprendizaje. Al participar los niños en actividades con sus pares incorporan las habilidades y perspectivas de su sociedad.

\section{1. ¿Qué se aprende en las familias?}

Las familias y no la familia ya que ahora hay una multiplicidad de realidades con respecto a su composición, sus límites, su estructura, funciones y roles por parte de sus integrantes (Gimeno, 2007), en las últimas décadas han tenido que vivir y sobrellevar grandes cambios, tal vez los más profundos y convulsivos, convirtiéndose para muchos en una auténtica revolución familiar (Valdivia, 2007). Sin embargo ésta sigue siendo para la mayoría de las personas una institución altamente significativa. Tiene un lugar relevante en la vida social y durante un buen tiempo cumple un papel muy importante para sus miembros. En este sentido queremos destacar la investigación desarrollada por el Programa de Educación en Valores de la Universidad Católica del Uruguay, en la misma surgen datos significativos: las personas consultadas en el transcurso de la investigación asignan el mayor nivel de importancia a la familia; para casi el total de la muestra (98.7\%) la familia es muy o bastante importante. Ante la pregunta sobre algunos cambios en nuestro estilo de vida que podrían ocurrir en un futuro próximo, la alternativa: "Que se de más importancia a la vida familiar" obtuvo el $94.1 \%$ de adhesión en la población general y el $92.7 \%$ en jóvenes (Da Costa, 2003).

La familia se transforma en la plataforma de lanzamiento para el proceso humanizador de todo sujeto, aunque no siempre logra alcanzar los objetivos (Buxarrais y Zeledón, 2007). La familia es el primer agente socializador para el individuo (LLopis y Llopis, 2003; Pérez Alonso-Geta, 2003) es el ámbito en el que la persona llega a este mundo, se muestra a los demás y en él se forma, es el lugar más influyente en la construcción de la personalidad y en el proceso de socialización (Gervilla, 2008). Se aprende entonces acerca del mundo en que vivimos, cómo son las relaciones entre las personas y se comienza a formar un sistema de valores personales y una identidad propia. La familia resulta en buena parte responsable de la estabilidad emocional de sus integrantes, tanto en la niñez como en la etapa adulta (Flaquer, 1998).

La familia como núcleo básico de socialización juega un papel muy relevante en la participación guiada de los individuos que integran dicho grupo. La interacción con otros en la familia, apoya, potencia su desarrollo. Los niños/as desarrollan destrezas en la medida que comprenden y participan de las actividades de su cultura. Dentro de la educación familiar y las prácticas educativas los padres intentan ir moldeando las conductas que entienden deseables y adecuadas en sus hijos. Esas 
acciones que llevan adelante los padres y que intentan prefijar a través del moldeado reciben el nombre de estilos educativos parentales (LLopis y LLopis, 2003).

\section{Estilos educativos parentales}

"Hablamos de estilos educativos a la forma de actuar, derivada de unos criterios, y que identifica las respuestas que los adultos dan a los menores ante cualquier situación cotidiana, toma de decisiones o actuaciones" (Comellas 2003, p. 12). Se habla de estilo por su permanencia y estabilidad a lo largo del tiempo, aunque pueden existir modificaciones (Climent, 2009). Cuando nos referimos a estilos educativos parentales, hay que tener en cuenta las tendencias globales de comportamiento, los estilos más frecuentes, ya que con ello no se pretende decir que los padres utilicen siempre las mismas estrategias con todos sus hijos ni en todas las situaciones, sino que los padres, escogen dentro de un marco más amplio y flexible los posibles estilos educativos (Ceballos y Rodrigo, 1998). Según Coloma, (1993) son esquemas prácticos que reducen las pautas educativas a unas pocas dimensiones básicas, que, cruzadas entre sí dan como resultado diversos tipos de educación parental.

Según Darling \& Steinberg (1993) los estilos educativos parentales son un conjunto de actitudes hacia los hijos que les son transmitidas y que en su totalidad crean un clima emocional en el cual se expresan las conductas de los padres. El estilo educativo "parental" es el contexto emocional (característica del padre) donde toman relevancia las conductas educativas específicas (González Tornaría, Vandemeulebroecke y Colpin, 2001).

Dentro de la conceptualización de estilos más importantes y en los que nos detendremos serán el "modelo de autoridad parental" de Baumrind (1966) y el propuesto por Maccoby \& Martín (1983), quienes reformulan la propuesta de Baumrind.

Desde hace varias décadas el concepto de estilo parental acuñado por Baumrind (1966) ha sido ampliamente utilizado en investigaciones acerca de las consecuencias de la socialización familiar sobre la competencia de niños y adolescentes. Baumrind (1966) propone tres tipos de estilos educativos parentales en función del grado de control que los padres ejercen sobre sus hijos: el estilo autoritario, el permisivo y el democrático.
Los padres que se manejan desde un estilo autoritario le dan una importancia mayor a la obediencia de los hijos, limitan, circunscriben la autonomía. Los padres que funcionan desde el estilo permisivo se encuentran en el extremo opuesto al estilo descripto anteriormente, no ejercen prácticamente ningún control y permiten el mayor grado de autonomía. Los que ejercen el estilo democrático son padres que se encuentran a mitad de camino entre los estilos mencionados anteriormente, intentan controlar el comportamiento de sus hijos, funcionando desde la razón y el razonar con ellos y no desde la imposición o la fuerza.

Por su parte, Maccoby \& Martin (1983) proponen cuatro estilos parentales a partir de dos dimensiones afecto/comunicación y control/ establecimiento de límites. El apoyo/afecto refiere al amor, a la aprobación, a la aceptación y a la ayuda que se les brinda a los hijos. De esta manera los hijos se sienten amados, aceptados, entendidos y con lugar para ser tenidos en cuenta. La dimensión control parental hace referencia al disciplinamiento que intentan conseguir los padres, de esta manera los padres controlan y/o supervisan el comportamiento de sus hijos y además velan por el cumplimiento de las normas establecidas por ellos mismos. A partir de éstas dimensiones se desarrollan cuatro estilos parentales, el autoritario, el permisivo, el democrático y el negligente. Diversos autores han desarrollado ésta tipología en estudios o investigaciones (Valdivia, 2010; López-Soler, Puerto, López-Pina y Prieto, 2009; Gervilla, 2008; Oliva et al., 2007; Ato, Galián y Huéscar, 2007; Oliva y Parra, 2004; Arranz, Bellido, Manzano, Martín y Olabarrieta, 2004; Pérez Alonso-Geta, 2003; LLopis y LLopis, 2003; Aguilar Ramos, 2002b; Musitu, Buelga, Lila y Cava, 2001; Palacios, 1999; Ceballos y Rodrigo, 1998, Coloma, 1993).

Los padres que ejercen el estilo democrático (afecto, control y exigencia de madurez) tienen hijos con un mejor ajuste emocional y comportamental. Son padres que estimulan la expresión de las necesidades de sus hijos, promueven la responsabilidad y otorgan autonomía (Torío López, Peña Calvo e Inda Caro, 2008). El estilo democrático tiene un impacto muy positivo en el desarrollo psicológico de los niños, manifiestan un estado emocional estable y alegre, una elevada autoestima y autocontrol (Arranz et al., 2004). En la adolescencia estos niños tienden a mostrarse con una elevada 
autoestima y desarrollo moral y social, además de un mejor rendimiento académico, (LópezSoler et al., 2009) menor conflictiva con sus padres y mayor bienestar psicológico (Arranz et al., 2004; Torío López, Peña Calvo e Inda Caro, 2008) Tienen un mayor interés por la educación, cuentan con un nivel de satisfacción más alto, así como también mayor confianza para el afrontamiento de nuevas situaciones y un mayor grado de independencia. Este estilo implica la combinación de tres elementos: el afecto y la comunicación familiar; el fomento de la autonomía por parte de los progenitores y el establecimiento de límites y la supervisión de la conducta de sus hijos (Oliva et al., 2007). Los chicos y chicas que perciben más afecto en sus padres y además tienen un mayor grado de comunicación con ellos, han demostrado un mejor desarrollo emocional, y un mejor ajuste conductual (Parra, Oliva y Sánchez-Queija, 2004). Ante la presencia de un monto de afecto importante por parte de los padres, se ha visto que las estrategias disciplinarias de los adultos fueron más eficaces, sobre todo en el ajuste de sus hijos e hijas (Oliva, Parra, Sánchez-Queija y López, 2007). Los padres con estilo democrático trasmiten afecto, brindan apoyo, promueven la comunicación, establecen reglas a nivel familiar, procuran su cumplimiento utilizando el razonamiento inductivo como forma de disciplinamiento, logran autonomía y cooperación en sus hijos, contando estos con una mayor probabilidad de ser sociables (Alonso y Román, 2005; Lila y Gracia, 2005).

Por otro lado, quienes han crecido y vivido en un ambiente negligente, presentan un sin número de problemas académicos, emocionales y conductuales. La falta de afecto, de supervisión y guía, de estos niños y adolescentes conlleva efectos muy negativos en el desarrollo de los mismos: sienten inseguridad e inestabilidad, son dependientes de los adultos, tienen dificultad de relación con sus pares, tienen baja tolerancia a la frustración. Son niños y adolescentes que pueden presentar conductas delictivas o abusivas. Es un estilo caracterizado por la indiferencia, la permisividad, la pasividad, la irritabilidad y la ambigüedad, no hay normas ni afecto (Montero Jiménez y Jiménez Tallón, 2009; Arranz, et al., 2004). Es un estilo que utiliza el castigo físico hacia los hijos como medida disciplinaria, predomina la falta de coherencia, mínimo control e implicación emocional (Arranz et al., 2004). En ésta categorización de estilos se encuentran los padres con estilos educativos permisivos y autoritarios.

Los niños, luego adolescentes que viven en hogares con estilo permisivo demuestran ser poco obedientes, tienen dificultad en la interiorización de valores, viven situaciones de agresividad en la familia, tienen baja autoestima, padecen de falta de confianza, tienen bajos niveles de control de sus impulsos, manifiestan dificultades a nivel conductual, como ser el consumo de sustancias y alcohol. Los padres promueven una importante autonomía en sus hijos, los liberan del control, evitan el uso de las restricciones y el castigo (Torío López, Peña Calvo e Inda Caro, 2008).

En cuanto a los hijos de padres con estilo educativo autoritario, sus problemas se plantean a nivel emocional, escaso apoyo, consolidan en los chicos una baja autoestima y una confianza en sí mismos deteriorada. Las estrategias educativas reprobativas y poco afectivas se relacionan con el consumo abusivo de alcohol en adolescentes (Pons y Berjano, 1997). En el caso de niños se ha relacionado el castigo físico recibido con problemas de impulsividad y agresión de estos hacia sus pares. (Arranz et al., 2004; Fuentes, 1999) Es un estilo que provoca una mayor inadaptación personal y social, no favoreciendo el ajuste personal ni social (García Linares, Pelegrina y Lendínez, 2002).

Los padres manejan importantes niveles de control restrictivo, bajos niveles de comunicación y afecto, los chicos funcionan con un fuerte control externo (Montero Jiménez y Jiménez Tallón, 2009). Los padres valoran la obediencia como una virtud, mantienen a sus hijos subordinados y restringen su autonomía. (Torío López, Peña Calvo e Inda Caro, 2008).

Los chicos se muestran tímidos, mínima expresión de afecto con sus pares, pobre interiorización de valores, son irritables, vulnerables a las tensiones y poco alegres (MacCoby \& Martin, 1983).

Estas características pueden llevarlos a que tengan tendencias a sentirse culpables y a padecer mayores niveles de depresión (Ato et. al, 2007). Es frecuente que cuando el control es muy severo estos chicos y chicas terminen rebelándose ante sus padres, especialmente al principio de la adolescencia, momento en el que suelen buscar una mayor libertad y autonomía (Figura 1). 


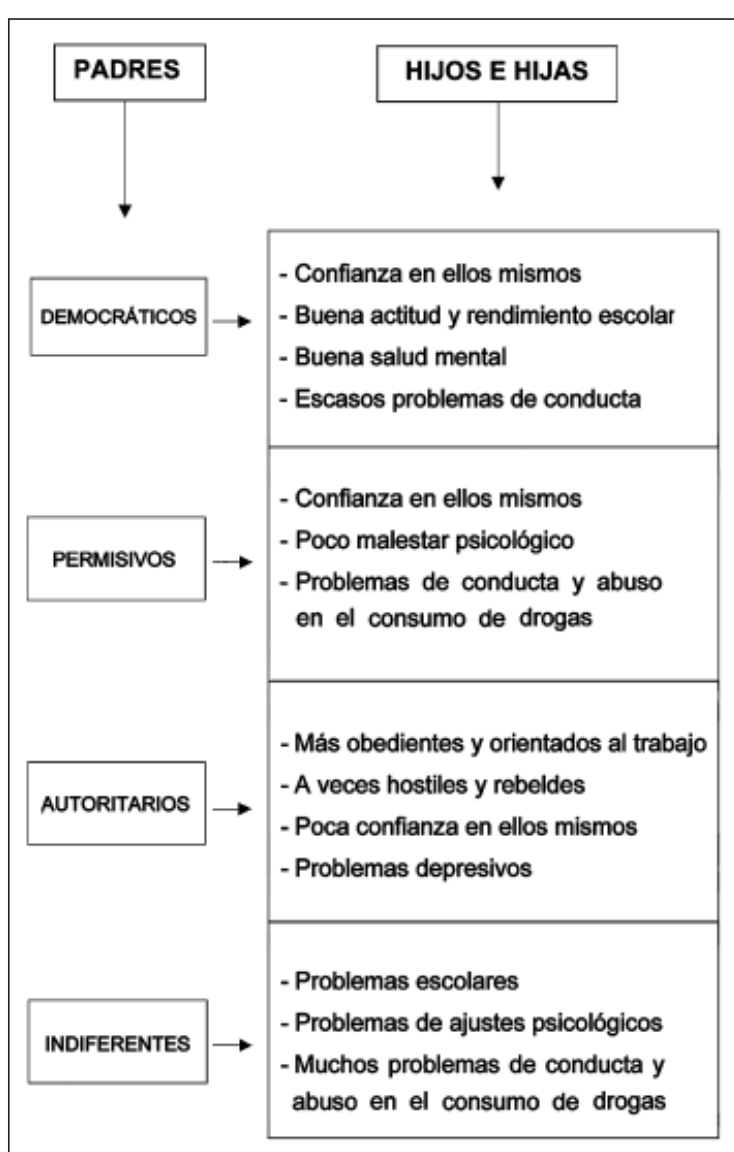

Nota: Síntesis de las investigaciones de (Lamborn et.al. 1991; Darling \& Steinberg, 1993). Tomado de Nuñez Cubero, L. como se citó en Gervilla, 2003).

Figura 1. Relación entre los estilos educativos paternos y las características de sus hijos adolescentes

\subsection{Sobre el estilo parental democrático}

Cabe manifestar que este estilo democrático es el que se ha considerado más adecuado para que los niños y adolescentes sean autocontrolados y estables psíquica y emocionalmente. Aunque un estudio reciente en España con una muestra representativa nacional de 1103 padres de niños y adolescentes de entre 6 y 14 años nos indica que, el estilo parental considerado más idóneo es el permisivo (Pérez Alonso-Geta, 2012).

De acuerdo al relevamiento realizado, la mayoría de los estudios han destacado una importante evidencia a favor del estilo democrático en todo tipo de cultura (Steinberg, 2001) y población de riesgo (Steinberg, Blatt-Einsengart \& Cauffman, 2006). Steinberg, Lamborn, Dor- busch \& Darling (1992) estudiaron el impacto que podría tener en 6400 adolescentes de entre 14 y 18 años el ejercicio parental democrático en su rendimiento académico. Se concluyó que los padres promueven y logran generar en los hijos un alto nivel académico y un fuerte compromiso con su escolaridad.

Finalmente, luego del recorrido realizado, hemos concluido que el estilo parental democrático es el más adecuado en el desarrollo de los niños y adolescentes. Sin embargo, y de acuerdo a nuestra experiencia en el trabajo con familias, se nos plantea alguna duda con respecto al término democrático, hemos escuchado críticas al término, a las relaciones democráticas en la vida familiar, a la igualdad de derechos o no que tienen los integrantes del núcleo familiar, al "laissez faire". Nosotros creemos que la idea de democracia ha confundido a muchos padres e hijos en relación al lugar que deben asumir y la forma en que deben relacionarse. Hay que tener claro que cuando se habla de estilo parental democrático en ningún momento se hace referencia al abandono de las exigencias y control, (Palacios, 1999) ni a la falta de la sanción cuando el no cumplimiento de las normas lo requiera. Tenemos la impresión de que en el imaginario social, en algunos estamentos sociales, estaría afincada la idea de que con un estilo parental democrático los niños o adolescentes pueden hacer en sus familias prácticamente lo que quieran.

En este sentido y para despejar cualquier duda o confusión es que nos parece más apropiado utilizar el término y la concepción propuesta desde la parentalidad positiva. Este estilo de parentalidad es un valioso recurso para la socialización, propone un protagonismo de padres, madres e hijos/as a la hora de la construcción de normas y valores familiares, apoyándose para eso en la negociación y adaptación conjunta. En este modelo se habla de contribución progresiva de los hijos al proceso de socialización, valiéndose del razonamiento y la reflexión para la construcción conjunta. Se menciona además la necesidad de ejercer responsablemente la autoridad por parte de los padres, preservando los derechos de los niños/ as (Rodrigo, Máiquez y Martin, 2010).

Creemos nosotros que ésta concepción se adapta mejor a este tiempo, a las posibilidades de los adultos y a las necesidades de los niños/ as y adolescentes. 


\section{Una alternativa posible: la Parentalidad Positiva}

La parentalidad positiva se refiere al comportamiento de los padres sustentado en el interés superior del niño (Asamblea General de las Naciones Unidas, 1989) desde el cual se promueve la atención, el desarrollo de sus capacidades, el ejercicio de la no violencia, ofreciendo el reconocimiento y la orientación necesaria sin dejar de incluir el establecimiento de los límites que permitan el pleno desarrollo del niño y el adolescente (Rodrigo et al, 2010).

Un padre positivo (Rodrigo et al., 2010) es el que atiende, potencia, guía y reconoce a sus hijos como personas con pleno derecho. La parentalidad positiva no es permisiva y requiere de la implementación de los límites necesarios para que los niños puedan desarrollarse plenamente.

El Consejo de Europa ha promovido la Recomendación Rec (2006) 19 sobre políticas de apoyo al ejercicio positivo de la parentalidad. Define el desempeño positivo del rol parental como el conjunto de conductas parentales que procuran el bienestar de los niños y su desarrollo integral desde una perspectiva de cuidado, afecto, protección, enriquecimiento y seguridad personal, de no violencia, que proporciona reconocimiento personal y pautas educativas e incluye el establecimiento de límites para promover su completo desarrollo, el sentimiento de control de su propia vida y puedan alcanzar los mejores logros tanto en el ámbito familiar como académico, con los amigos y en el entorno social y comunitario (Consejo de Europa, Comité de Ministros, 2006).

Se propone un control parental basado en el apoyo, el afecto, el diálogo, la cercanía y la implicación en la vida diaria de los niños y adolescentes. A diferencia del modelo de control del estilo autoritario por parte de los padres, la autoridad en la parentalidad positiva se encuentra basada en el respeto, la tolerancia, la comprensión de ambas partes y el diálogo que lleva a la construcción de acuerdos que permitan el crecimiento y desarrollo de las relaciones paterno-filiales.

\section{Principios básicos de la Parentalidad Positiva}

Siguiendo los aportes realizados por Rodrigo et al., (2010), quienes plantean algunos principios en forma de guía y orientación, de manera que si los adultos pretenden seguirlos, sientan que los puede ayudar en el mejor desarrollo de los niños y adolescentes propiciando su bienestar.

Vínculos afectivos cálidos: Funcionan como barrera de protección, de ser duraderos, generan aceptación y sentimientos positivos. En este sentido se podría promover el fortalecimiento de los vínculos afectivos en la familia a lo largo de su desarrollo.

Entorno estructurado: Aporta guía y orientación para el aprendizaje de normas y valores. Esto promueve la instalación de hábitos y rutinas con el fin de organizar las actividades diarias. Se ofrece al niño un sentimiento de seguridad a través de una rutina predecible y del establecimiento de los límites necesarios.

Estimulación y apoyo: Para el aprendizaje a nivel familiar y educativo formal con el fin de lograr una alta motivación y el desarrollo de sus capacidades. Esto supone conocer características y habilidades de sus hijos. Es importante compartir tiempo de calidad con ellos.

Reconocimiento: De sus relaciones, actividades y experiencias, del valor que ellos tienen, sobre sus preocupaciones y necesidades. Es vital nuestra comprensión y tener en cuenta sus puntos de vista. Sería importante escucharlos y valorarlos como sujetos con pleno derecho.

Capacitación: Apunta a potenciar el valor de los hijos e hijas, a que se sientan protagonistas, competentes, capaces de producir cambios e influir con su opinión o accionar a los demás. Es significativo el establecimiento de espacios de escucha, reflexión y explicaciones de los mensajes que llegan a la familia y a ellos.

Educación sin violencia: Descartar toda forma de castigo físico o psicológico. Eliminando de esta manera la posibilidad de que imiten modelos de interacción inadecuados, degradantes y violatorios de los derechos humanos. Elogiar su buen comportamiento, y ante su mal comportamiento reaccionar con una explicación y, si es necesario, con una sanción que no los violente (ni física, ni emocionalmente), como imponerles un "tiempo de reflexión", reparar los daños, reducir su dinero para gastos personales, etc.

El ejercicio de la parentalidad positiva requiere de apoyos para poder llevarla a cabo, en ese sentido una propuesta de apoyo a los padres se puede brindar desde un ámbito formal o informal a través de una formación de padres y madres de familia. El ámbito puede generarse en el centro educativo al que pertenecen y asisten 
sus hijos, en centros sociales y comunitarios, en clubes deportivos, en centros de salud, etc. Todo lugar que cuente con grupos constituidos de población infantil y adolescente y en la cual se pueda acceder a sus padres, madres o adultos significativos.

\section{La formaición de padres}

Vila (1998, p. 502) define la formación de padres como "un conjunto de actividades voluntarias de aprendizaje por parte de los padres que tiene como objetivo proveer modelos adecuados de prácticas educativas en el contexto familiar y/o modificar y mejorar prácticas existentes con el objeto de promover comportamientos en los hijos y las hijas que son juzgados positivamente y erradicar los que se consideran negativos". De alguna forma intenta promover y afirmar el ejercicio de las habilidades parentales existentes y por otro lado estimular y consolidar el desarrollo de nuevas capacidades con el objetivo de que los padres adquieran entonces las habilidades y competencias necesarias para el mejor desempeño de sus funciones (Trivette \& Dunst, 2005). La formación de padres y madres implica la posibilidad de analizar y reflexionar sobre pensamientos, comportamientos y emociones de la vida real. A través de la misma se intenta promover cambios a nivel cognitivo, emocional y comportamental en los propios padres (Máiquez et al., 2000). La propuesta sobre la formación de padres se encuentra basada en un enfoque que apunta a la optimización de capacidades y no solo a la compensación de deficiencias, partiendo del convencimiento de que la actuación de las madres y los padres en la crianza y educación de la infancia tiene un rol central y que es una tarea sumamente importante aunque muy complicada, para la que los padres no llegan preparados y además no reciben una formación. Las familias necesitan de una orientación, asesoramiento o entrenamiento en alguna etapa del ciclo evolutivo de sus hijos e hijas, ya sea por problemas considerados menores 0 por grandes dificultades que puedan surgir. La formación de padres, creemos nosotros, podría realizarse mediante un tipo de enseñanza en la que los padres tienen la oportunidad de conocer una amplia gama de experiencias variadas que puedan ser contrastadas con las suyas propias (Máiquez et al., 2000). Esto se consigue gracias al intercambio con otros padres o madres, embarcados en la misma tarea, promoviendo en los padres el sentirse activos y protagonistas de la tarea educativa y la intervención podría encaminarse hacia el desarrollo de la confianza en sus capacidades para educar a sus hijos (Máiquez et al., 2000). En línea con lo dicho anteriormente, Comellas (2009) manifiesta que el encuentro e intercambio entre personas y/o familias, facilita la conexión y el trasvase de experiencias y de propuestas, originando el enriquecimiento colectivo. De esta manera, las personas aprenden sobre los propios eventos de la vida familiar y van construyendo su conocimiento a partir de las situaciones y de sus teorías implícitas previas. Se aprende a ser padre y madre en la medida que se atraviesan experiencias, se enfrentan obstáculos y se superan o aparecen situaciones que llevan a los padres a improvisar, pero sin duda es fundamental "la reflexión conjunta de cómo se actúa, de qué queremos para nuestros hijos, de cómo vamos a afrontar su formación, qué se espera de nosotros" (Entrena y Soriano 2003, p. 146). Durante el año 2011 un equipo de investigación de la Facultad de Psicología de la UCU -González Tornaría, Capano, Scafarelli y Guerra (2012)-, Ilevaron adelante un proyecto de "Propuesta de Formación de Padres y Madres de Familia". Si bien no se evaluó la eficacia, los resultados de la investigación-acción, ratifican la importancia y pertinencia del trabajo con padres, así como de la metodología utilizada. Se realizaron veinticuatro talleres en seis instituciones católicas de Montevideo e Interior del país. Los objetivos de los talleres fueron: generar espacios de reflexión y participación entre los padres participantes; fomentar la creación de grupos de diálogo; fortalecer el vínculo institución educativa-familia. Los participantes fueron padres y madres $(n=130$ representaron el $20 \%$ del universo total de potenciales participantes), de alumnos adolescentes de primer y segundo año de secundaria.

La evaluación se realizó a través de cuestionarios cerrados para padres y referentes institucionales.

Aspectos relevantes: los padres participantes manifestaron la importancia que tiene para el desempeño de su rol la educación para padres. Además le dan trascendencia al hecho que la institución educativa apoye y acompañe dicho 
proceso. De los padres que contestaron el formulario se obtuvieron los siguientes resultados: el $95 \%$ de los mismos considera importante que se generaran espacios de reflexión, participación e intercambio con otros padres; el 95\% señala que se lograron diálogos y discusiones enriquecedoras con los otros padres; el 81\% de los padres participantes dicen haber notado algún cambio en el modo en que se relaciona con su hijo/a (o en su relacionamiento familiar); el $85 \%$ manifiesta que su vínculo con la institución educativa se ha fortalecido y el 95\% señala que ha sido una experiencia beneficiosa y/o productiva para sí, que volvería a participar de una experiencia similar y que recomendaría los talleres a otros padres (González Tornaría et al, 2012). La relevancia de los programas de formación de padres así como su eficacia quedan de manifiesto en las investigaciones llevadas a cabo entre otros, por Bartau y Etxeberría (2005). De los resultados preliminares que arroja la investigación surge que el programa cumple con su finalidad, proporciona estrategias y recursos educativos a los padres. Con el objetivo de que procuren la cooperación y responsabilidad de sus hijos en las tareas y vida familiar. A su vez Bartau (2007), desarrolla en profundidad los resultados del Programa Corresponsabilidad Familiar (COFAMI). Desarrollan estrategias educativas para padres y madres, con la finalidad de promover la colaboración y la responsabilidad de los chicos en el trabajo y la vida familiar. Martinez González y Pérez, (2004) y Martínez González, Pérez y Álvarez, (2006), nos refieren sobre las valoraciones hechas por los padres y madres en relación a la utilidad y eficacia de las actividades de formación, demandan conocer estrategias para poder ser más asertivos y consistentes al educar a sus hijos, etc.

Por otro lado Rodrigo, Máiquez, Martín y Byrne (2008), nos presentan el Programa de Apoyo Personal y Familiar. De la evaluación realizada con una variedad de instrumentos de medida relacionadas con los objetivos del programa, han podido constatar la eficacia del programa en términos de ganancias prepostest. Robles y Romero (2011), realizan un trabajo de revisión en diferentes bases de datos sobre la eficacia de los programas de entrenamiento para padres en un período de 20 años (1989-2009). En ese sentido los resultados fueron satisfactorios en la promoción de cambios positivos en la conducta de los niños, mejora en las interacciones entre padres e hi- jos y en las conductas y actitudes parentales. Su eficacia se comprueba en la mejora de la comunicación familiar, en la resolución de conflictos y en la disminución del estrés parental.

De la misma forma Graziano y Diament (1992) realizaron un recorrido por más de un centenar de estudios empíricos y concluyeron que el entrenamiento de padres tiene múltiples efectos favorables en los padres y sus hijos.

\section{Conclusiones}

Tanto los estilos educativos parentales, como el desarrollo y la socialización, han sido estudiados durante varias décadas por una cantidad importante de autores, sin duda como comentamos anteriormente los trabajos de mayor incidencia han sido entre otros los de (Baumrind, 1966; Maccoby \& Martin 1983; Darling \& Steinberg, 1993; Rogoff, 1993; Vigotsky, 1979). No obstante esto, creemos que existen otros autores que pueden aportarnos interesantes reflexiones, datos que nos permitan ahondar en la temática.

Dentro de la tipología creada para estilos educativos parentales la más interesante, la que conlleva a las consecuencias más positivas y saludables a la hora de educar a los niños y niñas resulta ser como hemos visto la desarrollada en el estilo democrático. De cualquier forma, si bien creemos en las posibilidades educativas que brinda dicho estilo, nosotros pensamos en base a nuestra experiencia que sería más adecuado -ya que evita malos entendidos y/o confusiones- hablar y proponer como el modelo de crianza a la parentalidad positiva. La parentalidad positiva se refiere al comportamiento de los padres sustentado en el interés superior del niño (Asamblea General de las Naciones Unidas, 1989), desde el cual se promueve la atención, el desarrollo de sus capacidades, el ejercicio de la no violencia, ofreciendo el reconocimiento y la orientación necesaria sin dejar de incluir el establecimiento de los límites que permitan el pleno desarrollo del niño y el adolescente. (Rodrigo et.al 2010). La parentalidad positiva es un valioso recurso para la socialización, propone un protagonismo de padres, madres e hijos/as a la hora de la construcción de normas y valores familiares, apoyándose para eso en la negociación y adaptación conjunta. En este nuevo modelo se habla de contribución progresiva de los hijos al proceso de socialización, valiéndose del ra- 
zonamiento y la reflexión para la construcción conjunta. Se menciona además la necesidad de ejercer responsablemente la autoridad por parte de los padres, preservando los derechos de los niños/as. (Rodrigo et.al 2010).

Una forma de apoyar y potencializar para poner en práctica la parentalidad positiva es a través de la formación de padres y madres, en este sentido hay una multiplicidad de experiencias en diferentes partes, durante las últimas décadas. Las investigaciones, proyectos o programas de formación, entrenamiento, capacitación u orientación de padres (Bartau y Etxeberría, 2005; Bartau, 2007; Rodrigo et al., 2008; Gervilla, 2008; Entrena y Soriano, 2003; Vila, 1998; Máiquez et al., 2000; Martinez González y Pérez, 2004; Martínez González, 2009; Martínez González, et al. 2006; González Tornaría et al., 2012), coinciden en resaltar la importancia en la realización de estas experiencias, algunas de las investigaciones mencionadas plantean empíricamente la eficacia de las mismas, otros centran su relato en la satisfacción de los participantes con los programas, algunos otros toman en cuenta ambas variables, pudiéndose además realizar desde diferentes ámbitos (el educativo, el sanitario y el comunitario), así como desde el sector público o privado.

En general ser padres es algo para lo que no se educa a las personas, se va aprendiendo en la marcha, en la medida que se enfrentan los problemas, que se tiene que improvisar una salida ante una dificultad. Es en ese marco que tiene sentido la formación de padres, se puede mejorar el desempeño de los adultos en su quehacer cotidiano, permitiendo el mejor desarrollo físico, psicológico y social de niños $\mathrm{y}$ adolescentes.

\section{Referencias}

AguilarRamos, M.C.(2002a). FamiliayEscuelaanteunmundoen cambio. Revista Contextos de Educación, (V), 202-215.

Aguilar Ramos, M. C. (2002b). Educación Familiar: una propuesta disciplinar y curricular España: Aljibe

Alonso, J., y Román, J. M. (2005). Prácticas educativas familiares y autoestima. Psicothema, 17(1), 76-82.

Arranz, E., Bellido, A., Manzano, A., Martín, J. L., y Olabarrieta, F. (2004). Contexto familiar y desarrollo psicológico durante la infancia. En E. Arranz (Coord.), Familia y desarrollo psicológico. (pp. 70-95) Madrid: Pearson Educación

Asamblea General de las Naciones Unidas (1989). Convención sobre los Derechos del Niño. Recuperado de http://www.gobernacion.gob.mx/work/models/SEGOB/ Resource/689/1/images/Convencion_sobre_los_derechos_del_nino.pdf
Ato, E., Galián, Ma. Dolores., y Huéscar, E. (2007). Relaciones entre estilos educativos, temperamento $y$ ajuste social en la infancia: Una revisión. Anales de Psicología, 23(1), 33-40

Bartau, I. (2007). Educando en Igualdade. Supervivencia Doméstica: o exercicio da corresponsabilidade. Educar compartiendo las tareas familiares. VIII Xornada Gallega de Nais e Pais. Servizo Galego de Igualdade Xunta de Galicia. Santiago de Compostela.

Bartau, I., y Etxeberría, J. (2005). El programa corresponsabilidad familiar (COFAMI): Organización e Intervención en el País Vasco. Revista Electrónica Iberoamericana sobre Calidad, Eficacia y Cambio en Educación, 3(1), 586-593.

Baumrind, D. (1966). Effects of Authoritative Parental Control on Child Behavior. Child Development, 37(4), 887-907.

Bersabé, R., Fuentes, M., y Motrico, E. (2001). Análisis psicométricos de dos escalas para evaluar estilos educativos parentales. Psicothema 13 (4) 678-684.

Bronfenbrenner, U. (1987). La ecología del desarrollo humano. España: Paidós

Buxarrais, M.R., y Zeledón, M. P. (2007). ¿Por qué y para qué educar en valores democráticos desde el ámbito familiar? En M. Buxarrais, y M. Zeledón (Coords.), Las familias y la educación en valores democráticos. Retos y perspectivas actuales. (pp.159-183) Barcelona: Claret

Ceballos, E., y Rodrigo, M.J. (1998). Las metas y estrategias de socialización entre padres e hijos. En M. J. Rodrigo y J. Palacios (Comp.), Familia y desarrollo humano. (pp.225-243). Madrid: Alianza.

Climent, G. (2009). Voces, silencios y gritos: Los significados del embarazo en la adolescencia y los estilos parentales educativos. Revista Argentina de Sociología, (12/13), 186-213.

Coloma, J. (1993). Estilos Educativos Paternos. En J. M. Quintana (Coord.), Pedagogía Familiar. (pp. 45-58) Madrid: Narcea.

Comellas, M.J. (Junio, 2003). Criterios educativos básicos en la infancia como prevención de trastornos. Recuperado de http://www.avpap.org/documentos/comellas.pdf

Comellas, M.J. (2009). Familia y escuela: compartir la educación. Barcelona: Graó.

Consejo de Europa, Comité de Ministros (13 de diciembre de 2006). Recomendación Rec (2006)19 del Comité de Ministros a los Estados Miembros sobre políticas de apoyo al ejercicio positivo de la parentalidad. Recuperado de http://www.msps.es/politicaSocial/ familiasInfancia/docs/recomendacion.pdf

Da Costa, N. (Coord.) (2003). Los valores de los uruguayos. Montevideo: Universidad Católica del Uruguay.

Darling, N., \& Steinberg, L.(1993). Parenting styles as context: an integrative model. Psychological Bulletin, 113, 487-496

DeMause, L.L. (1994). Historia de la Infancia. Madrid: Alianza Editorial

Entrena, M., y Soriano, A. (2003). Escuelas de Padres. En E. Gervilla (Comp.), Educación Familiar. Nuevas relaciones humanas y humanizadoras. (pp.143-154) Madrid: Narcea.

Flaquer, L. (1998). El destino de la familia. Barcelona: Ariel.

Fuentes, M. J. (1999) Los grupos, las interacciones entre compañeros y las relaciones de amistad en la infancia y adolescencia. En F. López, I. Etxebarria, J.M. Fuentes y J.M. Ortiz (Coords.), Desarrollo Afectivo y Social. (pp. 151-180) España: Pirámide 
García Linares, M. C., Pelegrina, S., y Lendínez, J. (2002). Los estilos educativos de los padres y la competencia psicosocial de los adolescentes. Anuario de Psicología, 33, 79-95.

García Méndez, E. (1998). Infancia: de los derechos, de la justicia. Buenos Aires: Ediciones del Puerto

Gervilla, A. (2008). Familia y Educación Familiar; Conceptos clave, situación actual y valores. Madrid: Narcea

Gimeno, A. (2007). Las familias en la sociedad de la información y de la diversidad. En M. Buxarrais y M. Zeledón (Coords.), Las familias y la educación en valores democráticos. Retos y perspectivas actuales. (pp.99-126) Barcelona: Claret.

González Tornaría, M. del L. (2007). El reto de las familias en los procesos de educación en valores democráticos. En M. Buxarrais y M. Zeledón (Coords.), Las familias y la educación en valores democráticos. Retos y perspectivas actuales. (pp.159-183) Barcelona: Claret.

González Tornaría, M. del L., Capano, A., Scafarelli, L., Guerra, A. (2012). Presentación de una Propuesta de Formación de Padres y Madres de Familia. PSICO 3(3), 408-416.

González Tornaría, M. del L., Vandemeulebroecke, L., y Colpin, H. (2001). Pedagogía Familiar. Aportes desde la teoría y la investigación. Uruguay: Trilce.

Graziano, A. M., \& Diament, D. M. (1992). Parent behavioral training: An examination of paradigm. Behavior Modification, 16(1), 3-38

Hernández Prados, Ma. de los A. y López, H. (2006). Análisis del enfoque actual de la cooperación padres y escuela. Aula Abierta, 87, 3-26.

Kuhn, T. (1988). La estructura de las revoluciones científicas. México: Fondo de Cultura Económica. (Trabajo original publicado en 1962)

Lila, M., y Gracia, E. (2005). Determinantes de la aceptación-rechazo parental. Psicothema, 17(1), 107-111.

López-Soler, C., Puerto, J., López-Pina, J. y Prieto, M. (2009). Percepción de los estilos educativos parentales e inadaptación en menores pediátricos. Anales de psicología, 25(1), 70-77

LLopis, D. y LLopis, R. (2003). Estilos educativos parentales y relaciones sociales en adolescentes. FAM, 27, 53-70

Maccoby, E.E., y Martín, J. A. (1983). Socialization in the context of the family: Parent-child interaction. En E. M. Hetherington \& P.H. Mussen (Eds), Handbook of child psychology: Socialization, personality and social development Vol.4 ( pp.1-101). New York: Wiley

Máiquez, M. L., Rodrigo, M. J., Capote, C., y Vermaes, I. (2000). Aprender en la vida Cotidiana. Un programa experiencial para padres. Madrid: Visor.

Martínez González, R. A. (2009). Programa-Guía para el Desarrollo de Competencias Emocionales, Educativas y Parentales. Madrid: Ministerio de Sanidad y Política Social.

Martínez González, R. A., y Pérez, M. (2004). Evaluación e intervención educativa en el campo familiar. REOP, 15(1), 89-104.
Martínez González, R. A. , Pérez, M. y Álvarez, L. (2006). Estrategias para prevenir y afrontar conflictos en las relaciones familiares (padres e hijos). (Informe de Investigación). Recuperado de http://www.observatoriodelainfancia.msssi.gob.es/ documentos/Estrategias-prevenir-afrontar-conflictos. pdf

Montero Jiménez, M. \& Jiménez Tallón, M. A. (2009). Los estilos educativos parentales y su relación con las conductas de los adolescentes. Familia, 39, 77-104

Musitu, G., Buelga, S., Lila, M., y Cava, M.J. (2001). Familia y Adolescencia. España: Sintesis

Musitu, G., y Cava, M.J. (2001). La familia y la educación. Barcelona: Octaedro.

Oliva, A., Hidalgo, V., Martín, D., Parra, A., Ríos, M., y Vallejo, R. (2007). Programa de Apoyo a Madres y Padres de Adolescentes. Recuperado de http:// etfgranada.blogspot.com/2012/09/programa-deapoyo-madres-y-padres-de.html

Oliva, A. y Parra, A. (2004). Contexto familiar y desarrollo psicológico durante la adolescencia. En E. Arranz (Coord), Familia y desarrollo psicológico. (pp. 96-123) Madrid: Pearson Educación

Oliva, A., Parra, A. y Arranz, E. (2008). Estilos relacionales parentales y ajuste adolescente. Infancia y Aprendizaje, 31(1), 93-106

Oliva, A., Parra, A., Sánchez-Queija, I., y López, F. (2007). Estilos educativos materno y paterno: Evaluación y relación con el ajuste adolescente. Anales de Psicología, 23(1), 49-56

Palacios, J. (1999). La familia y su papel en el desarrollo afectivo y social. En F. López, I. Etxebarria, M.J. Fuentes, y M.J. Ortiz, (Coords) Desarrollo Afectivo y Social (pp. 267-281) Madrid: Pirámide.

Parada, J.L. (2010). La educación familiar en la familia del pasado, presente y futuro. Educatio Siglo XXI, 28(1), 17-40.

Parra, A., Oliva, A. y Sánchez-Queija, I. (2004). Evolución y determinantes de la autoestima durante los años adolescentes. Anuario de Psicología, (35) 331-346.

Pérez Alonso-Geta, P. (2012). La socialización parental en padres españoles con hijos de 6 a 14 años. Psicothema, 24(3), 371-376.

Pérez Alonso-Geta, P. (2003). Crianza y estilos familiares de educación. En E. Gervilla (Coord.), Educación Familiar. Nuevas relaciones humanas y humanizadoras (pp. 65-80) Madrid: Narcea.

Pons Diez, J. y Berjano, E. (1997). Análisis de los estilos parentales de socialización asociados al abuso de alcohol en adolescentes. Psicothema, 9 (3) 609-617.

Robles, Z., y Romero, E. (2011). Programas de entrenamiento para padres de niños con problemas de conducta: una revisión de su eficacia. Anales de Psicología, 27(1), 86-101.

Rodrigo, M. J., Máiquez, M.L., y Martín, J. (2010). Parentalidad positiva y políticas locales de apoyo a las familias. Orientaciones para favorecer el ejercicio de las responsabilidades parentales desde las corporaciones locales. Madrid: FEMP 
Rodrigo, M.J., Maiquez, M.L., Martín, J., y Byrne, S. (2008). Preservación familiar. Un enfoque positivo para la intervención con familias. Madrid: Pirámide.

Rogoff, B. (1993). Aprendices del pensamiento. El desarrollo cognitivo en el contexto social. Barcelona: Paidos.

Steinberg, L. (2001). We know some things: parentadolescent relationships in retrospect and prospect. Journal of Research on Adolescence, 11, 1-19.

Steinberg, L., Blatt-Einsengart, I., \& Cauffman, E.; (2006). Patterns of competence and adjustment among adolescents from authoritative, authoritarian, indulgent, and neglectful homes: A replication in a sample of serious juvenile offenders. Journal of Research on Adolescence, 16, 47-58.

Steinberg, L., Lamborn, S., Dorbusch, S., \& Darling, N. (1992). Impact of Parenting Practices on Adolescent Achievement: Authoritative Parenting, School Involvement, and Encouragement to Succeed. Child Development, 63, 1266-1281.

Torío López, S., Peña Calvo, J.V. e Inda Caro, M. (2008). Estilos de educación familiar. Psicothema, Vol. 20 (1), 62-70
Torío López, S., Peña Calvo, J.V. y Rodríguez Menéndez, M del C. (2008). Estilos Educativos Parentales. Revisión Bibliográfica y Reformulación Teórica. Teoría Educativa, (20), 151-178.

Trivette, C. \& Dunst, C. (2005). "Community-based parent support programs". En R. Tremblay, R. Barr, \& Peters, R (Eds.), Encyclopedia on Early Childhood Development. Montreal, Quebec: Centre of Excellence for Early Childhood Development.

Valdivia, M. del C. (2007). Nuevos modelo de familia. En M. R. Buxarrais y M. P. Zeledón (Coords.), Las familias y la educación en valores democráticos. Retos y perspectivas actuales. (pp.35-70) Barcelona: Claret

Valdivia, M. del C. (2010). Madres y padres competentes. Claves para optimizar las relaciones con nuestros hijos e hijas. España: Graó.

Vigotsky, L. (1979). El desarrollo de los procesos psicológicos superiores. Barcelona: Grijalbo.

Vila, I. (1998). Intervención psicopedagógica en el contexto familiar. In Rodrigo, M.J. \& Palacios, J. (Comp), Familia y desarrollo humano. (pp.501-519) Madrid: Alianza.

Para citar este artículo:

Capano, A., y Ubach, A. (2013). Estilos parentales, Parentalidad Positiva y formación de padres.

Ciencias Psicológicas VII (1): 83 -95. 